\title{
COUPLED HEAT AND MASS TRANSFER BY MHD NATURAL CONVECTION OF MICROPOLAR FLUID ABOUT A TRUNCATED CONE IN THE PRESENCE OF RADIATION AND CHEMICAL REACTION
}

\author{
Ali J. Chamkha' ${ }^{1}$ S.M.M. EL-Kabeir ${ }^{2,3}$, A.M. $\operatorname{Rashad}^{3}$ \\ ${ }^{1}$ Manufacturing Engineering Department, The Public Authority for Applied Education and Training, Shuweikh 70654, \\ Kuwait, Email: achamkha@yahoo.com \\ ${ }^{2}$ Department of Mathematics, Salman bin Abdulaziz University, College of Science and Humanity Studies, AL-Kharj, Saudi \\ Arabia, Email: elkabeir@yahoo.com \\ ${ }^{3}$ Department of Mathematics, Aswan University, Faculty of Science, Aswan, 81528, Egypt, Email: am_rashad@yahoo.com
}

\begin{abstract}
:
An analysis is performed to study the thermal radiation and chemical reaction effects on coupled heat and mass transfer by MHD natural convective boundary-layer flow of a micropolar fluid over a permeable truncated cone with variable surface temperature and concentration. A suitable set of dimensionless variables is used to transform the governing equations of the problem into a nonsimilar form. The resulting non-similar equations have the property that they reduce to various special cases previously considered in the literature. An adequate and efficient implicit, tri-diagonal finite difference scheme is employed for the numerical solution of the obtained equations. Various comparisons with previously published work are performed and the results are found to be in excellent agreement. A representative set of numerical results for the velocity, microrotation, temperature and concentration profiles as well as the local skin-friction coefficient, local wall couple stress, local Nusselt number and the local Sherwood number is presented graphically for various parametric conditions and discussed.
\end{abstract}

Keywords: Heat and transfer, micropolar fluid, thermal radiation, chemical reaction, MHD natural convection

\section{NOMENCLATURE}

$a_{r} \quad$ Rosseland mean extinction coefficient

$B \quad$ spin gradient viscosity parameter

$B_{o} \quad$ magnetic induction

$c \quad$ concentration

$c_{p} \quad$ specific heat at constant pressure

$C_{f} \quad$ local skin-friction coefficient, $2 \mathrm{v}(\partial \mathrm{u} / \partial \mathrm{y})_{\mathrm{y}=0} / \mathrm{U}_{\mathrm{r}}^{2}$

D mass diffusivity

$f \quad$ dimensionless stream function

$f_{o} \quad$ wall mass transfer coefficient, $\mathrm{v}_{0} / v\left(\mathrm{x}_{0}^{4} / \mathrm{Gr}_{\mathrm{x}_{0}}\right)^{1 / 4}$

$g \quad$ microrotation

$g^{*} \quad$ gravitational acceleration

$\mathrm{Gr}_{\mathrm{x}}{ }^{*} \quad$ local Grashof number, $\mathrm{g} \cos \gamma \beta_{\mathrm{T}}\left(\mathrm{T}_{\mathrm{w}}-\mathrm{T}_{\infty}\right)\left(\mathrm{x}^{*}\right)^{3} / \mathrm{v}^{2}$

$h \quad$ local heat transfer coefficient

$h_{m} \quad$ local mass transfer coefficient

j microinertia density

$K \quad$ micropolar fluid vortex viscosity parameter

$k \quad$ thermal conductivity

$k_{r} \quad$ dimension of chemical reaction

$H a$ Hartmann number, $\left(\sigma^{*} \mathrm{~B}_{\mathrm{o}}^{2} /(\rho v)\left(\mathrm{x}_{\mathrm{o}}^{4} / \mathrm{Gr}_{\mathrm{x}_{\mathrm{o}}}\right)^{1 / 2}\right)^{1 / 2}$

$N$ component of the microrotation vector normal to $\mathrm{x}-\mathrm{y}$ plane
$N u_{x^{*}} \quad$ local Nusselt number, $\mathrm{hx}^{*} / \mathrm{k}$

Pr Prandtl number, $v / \alpha$

$r \quad$ local radius of the truncated cone

$r_{t} \quad$ surface temperature parameter, $\mathrm{T}_{\mathrm{w}} / \mathrm{T}_{\infty}$

$R_{d} \quad$ radiation-conduction parameter, $4 \sigma \mathrm{T}_{\infty}^{3} /\left[\mathrm{k}\left(\mathrm{a}_{\mathrm{r}}+\sigma_{\mathrm{s}}\right)\right]$

Sc $\quad$ Schmidt number, $v / \mathrm{D}$

$S h_{x^{*}} \quad$ local Sherwood number, $\mathrm{h}_{\mathrm{m}} \mathrm{x}^{*} / \mathrm{D}$

$T$ temperature

$u \quad$ velocity component in the $\mathrm{x}$-direction

$U_{r} \quad$ reference velocity, $\left[g \cos \gamma \beta_{\mathrm{T}}\left(\mathrm{T}_{\mathrm{w}}-\mathrm{T}_{\infty}\right) \mathrm{x}^{*}\right]^{1 / 2}$

$v \quad$ velocity component in the $\mathrm{y}$-direction

$v_{\mathrm{o}} \quad$ wall suction or injection velocity

$x \quad$ streamwise coordinate

$x_{\mathrm{o}} \quad$ distance of the leading edge of truncated cone measured from the origin

$x^{*} \quad$ distance measured from the leading edge of the truncated cone, $\mathrm{x}-\mathrm{x}_{\mathrm{o}}$

$y$ transverse coordinate

\section{Greek Symbols}

$\alpha \quad$ thermal diffusivity

$\beta_{c} \quad$ coefficient of concentration expansion 


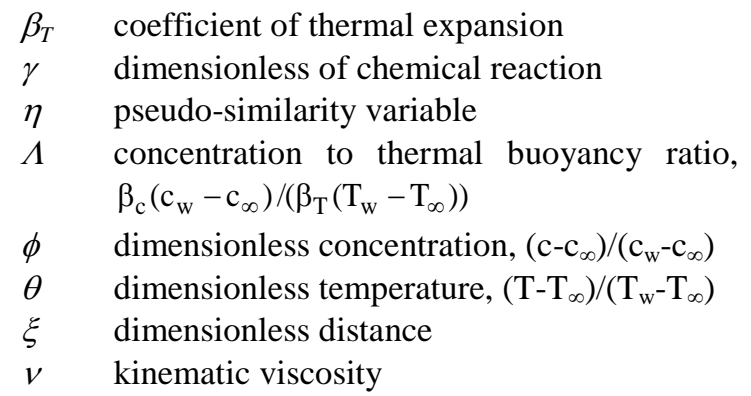

$\begin{array}{ll}\Omega & \text { half angle of the truncated cone } \\ \rho & \text { density } \\ \sigma & \text { Stefan-Boltzmann constant } \\ \sigma^{*} & \text { electrical conductivity } \\ \sigma_{s} & \text { scattering coefficient } \\ \psi & \text { stream function } \\ \text { Subscripts } \\ w & \text { condition at the wall } \\ \infty & \text { condition at infinity }\end{array}$

\section{Introduction}

An important categories of non-Newtonian fluids include viscoelastic fluids which show partial elastic recovery on removal of the deforming stress, pseudoplastic fluids which exhibit a yield stress feature and represent accurately for example heavy crude oils with a high wax content, and Bingham plastics which do not flow until a certain critical yield stress is attained. Micropolar fluids are fluids with microstructure belonging to a class of fluids with nonsymmetrical stress sensor referred to as polar fluids. Physically, they represent fluids consisting of randomly oriented particles suspended in a viscous medium, and this fluid is the fluid with internal structures in which coupling between the spin of each particle and the macroscopic velocity field is taken into account. It is a hydrodynamical framework suitable for granular systems which consist of particles with macroscopic size, and they are important to engineers and scientists working with hydrodynamic-fluid problems and phenomena. Examples of the latter include metallic oxides, clay-water suspensions (e.g. river sediments), dental creams, paints and thickened hydrocarbon greases. The theory about micropolar fluids and the momicropolar fluids has been developed by Erigen $(1966,1972)$, and excellent reviews about the applications of micropolar fluids have been written by Airman et al. (1973,1974). EL-Kabeir (2004) analyzed radiative effects on forced convection flows in micropolar fluids with variable viscosity. EL-Kabeir (2005) discussed the Hiemenz flow of a micropolar viscoelastic fluid in hydromagnetics. EL-Kabeir and Modather (2007) have also presented a study for chemical reaction, heat and mass transfer on MHD flow over a vertical isothermal cone surface in micropolar fluids with heat generation/absorption. Cheng (2008) presented a boundary-layer analysis about the natural convection heat transfer near a vertical truncated cone with power-law variation in surface temperature in a micropolar fluid. Chang (2008) discussed numerical simulation for natural convection of micropolar fluids flow along slender hollow circular cylinder with wall conduction effect. Rebhi et al. (2009) studied combined effect of heat generation or absorption and first-order chemical reaction on micropolar fluid flows over a uniformly stretched permeable surface. Modather et al. (2009) considered the effect of chemical reaction on the heat and mass transfer of micropolar fluids in a saturated porous medium over an infinite moving permeable plate. EL-Kabeir et al. (2010) investigated coupled heat and mass transfer on MHD stagnation-point flow of a power-law fluid towards a stretching surface with thermal radiation and chemical reaction effects. Pal and Chatterjee (2010) studied the heat and mass transfer in MHD non-Darcian flow of a micropolar fluid over a stretching sheet embedded in a porous media with non-uniform heat source and thermal radiation. Kishan and Amrutha (2011) studied the effects of thermal stratification and chemical reaction on MHD flow with heat and mass transfer over a stretching surface with heat source. EL-Kabeir et al. (2011) discussed the heat transfer in a micropolar fluid flow past a permeable continuous moving surface. Rashidi et al. (2011) studied the analytic approximate solutions for heat transfer of a micropolar fluid through a porous medium with radiation. Pal (2011) analyzed the combined effects of non-uniform heat source/sink and thermal radiation on heat transfer over an unsteady stretching permeable surface. The effects of Joule-heating, chemical reaction and thermal radiation on unsteady MHD natural convection from a heated vertical porous plate in a micropolar fluid are analyzed by Chamkha et al. (2011). Rashad (2013) studied the effect of thermal radiation on unsteady MHD flow of a rotating fluid from stretching surface in porous medium.

Hence, the purpose of the present work is to study the effects of chemical reaction and thermal radiation on the coupled heat and mass transfer by MHD natural convection boundary-layer flow of a micropolar fluid over a permeable truncated cone with variable surface temperature and concentration. The governing boundary-layer equations have been transformed to a non-similar form, and these have been solved numerically. The effects of magnetic field, thermal radiation, chemical reaction, injection or suction and power-law variations of both the wall temperature and concentration on the velocity, microrotation, temperature and concentration profiles as well as the local skin-friction coefficient, local couple stress, local Nusselt number and local Sherwood number have been shown graphically and discussed. 


\section{Governing Equations}

Consider the problem of coupled heat and mass transfer by MHD natural convection, boundary layer flow of an electrically-conducting, optically dense and micropolar fluid about a truncated permeable cone in the presence of radiation and chemical reaction effects. Figure 1 shows the flow model and the physical coordinate system. The origin of the coordinate system is placed at the vertex of the full cone, where $x$ is the coordinate along the surface of the cone measured from the origin and $y$ is the coordinate normal to the surface, respectively. The cone surface is maintained at a variable temperature $T_{w}(x)$ and a variable concentration $c_{w}(x)$ and the ambient temperature and concentration far away from the surface of the cone $T_{\infty}$ and $c_{\infty}$ are assumed to be uniform. For $T_{w}>T_{\infty}$ and $c_{w}>c_{\infty}$, an upward flow is induced as a result of the thermal and concentration buoyancy effects. $T_{w}(x)$ and $c_{w}(x)$ are assumed to vary as power-law functions of the distance along the cone surface $x$. Fluid suction or injection is imposed at the cone surface. A uniform magnetic field is applied in the $y$-direction normal to the flow direction. The magnetic Reynolds number is assumed to be small so that the induced magnetic field is neglected. In addition, the Hall effect and the electric field are assumed negligible. The small magnetic Reynolds number assumption uncouples the Navier-Stokes equations from Maxwell's equations. A first-order homogeneous chemical reaction is assumed to take place in the flow. The flow is assumed laminar, steady and all of the micropolar fluid properties are assumed to be constant except for the density variation in the buoyancy force term. By invoking all of the boundary layer, Boussineq and Rosseland diffusion approximations (see, for instance, Yih (1999) and Chamkha (2001)) the governing equations for this investigation can be written as;

$$
\begin{aligned}
& \frac{\partial(r u)}{\partial x}+\frac{\partial(r v)}{\partial y}=0 \\
& u \frac{\partial u}{\partial x}+v \frac{\partial u}{\partial y}=\left(\frac{\mu+k}{\rho}\right) \frac{\partial^{2} u}{\partial y^{2}}+\frac{k}{\rho} \frac{\partial N}{\partial y}+g^{*} \beta_{T}\left(T-T_{\infty}\right) \cos \Omega+g^{*} \beta_{c}\left(c-c_{\infty}\right) \cos \Omega-\frac{\sigma^{*} B_{o}^{2}}{\rho} u \\
& u \frac{\partial N}{\partial x}+v \frac{\partial N}{\partial y}=\frac{\gamma}{\rho j} \frac{\partial^{2} N}{\partial y^{2}}-\frac{k}{\rho j}\left(2 N+\frac{\partial u}{\partial y}\right) \\
& u \frac{\partial T}{\partial x}+v \frac{\partial T}{\partial y}=\alpha \frac{\partial^{2} T}{\partial y^{2}}+\frac{16 \sigma}{3\left(a_{r}+\sigma_{s}\right) \rho c_{p}} \frac{\partial}{\partial y}\left(T^{3} \frac{\partial T}{\partial y}\right) \\
& u \frac{\partial c}{\partial x}+v \frac{\partial c}{\partial y}=D \frac{\partial^{2} c}{\partial y^{2}}-k_{r}\left(c-c_{\infty}\right)
\end{aligned}
$$

where all of the parameters are defined in 'Nomenclature'.

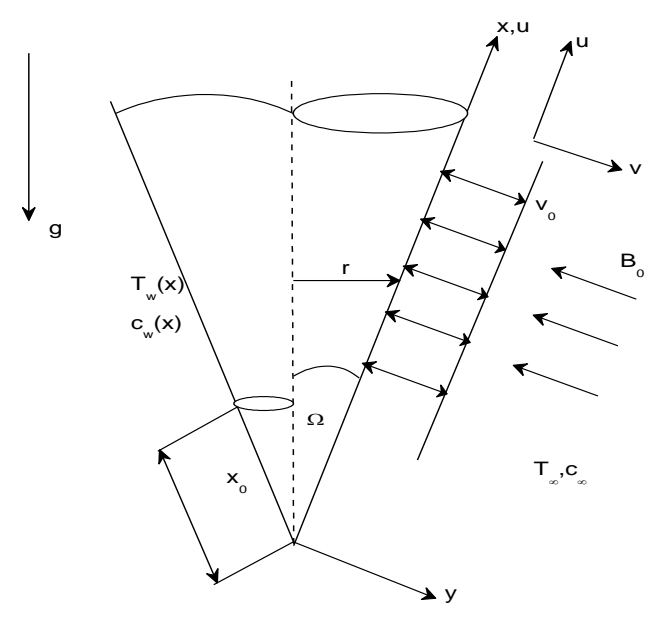

Figure 1: Problem Schematics and Coordinate System

The boundary and ambient conditions for this problem can be written as:

$$
\begin{aligned}
& y=0: u=0, v=-v_{o}, N=-m \frac{\partial u}{\partial y}, T=T_{w}(x)=T_{\infty}+a_{1}\left(x-x_{o}\right)^{n}, c=c_{w}(x)=c_{\infty}+b_{1}\left(x-x_{o}\right)^{n} \\
& y \rightarrow \infty: \quad u \rightarrow 0, \quad N \rightarrow 0, T \rightarrow T_{\infty}, \quad c \rightarrow c_{\infty},
\end{aligned}
$$


where $v_{\mathrm{o}}$ represents the transpiration velocity of fluid through the surface of the cone or the permeability of the porous surface where its sign indicates suction or withdrawal of fluid $(>0)$, blowing or injection of fluid $(<0)$ and $(=0)$ is the case when the cone surface is impermeable. Here $a_{1}, b_{1}$ and $n$ are constants.

The boundary layer remains thin because it grows less quickly than does the radius of the cone. The local radius to a point in the boundary layer, therefore, can be replaced by the radius of the truncated cone $r$, i.e., $r=x \sin \Omega$. Eqs. (1)-(7) are valid in $x_{0}<x<\infty$. The continuity equation may be satisfied by introducing a stream function by the customary definition. We follow the work of Rees and Bassom (1996) by assuming that $\gamma^{*}$ is given by,

$\gamma^{*}=\left(\mu+\frac{k}{2}\right) j=\mu\left(1+\frac{K}{2}\right) j, \quad K=\frac{k}{\mu}$

The governing equations and boundary conditions can be made dimensionless by introducing the stream function such that

$r u=\frac{\partial \psi}{\partial y}, \quad r v=-\frac{\partial \psi}{\partial x}$,

and using the following dimensionless variables

$$
\begin{aligned}
& \xi=\frac{x-x_{o}}{x_{o}}=\frac{x^{*}}{x_{o}}, \quad \eta=\frac{y}{x^{*}}\left(G r_{x} .\right)^{1 / 4}, \quad G r_{x} \cdot=g^{*} \cos \Omega \beta_{T}\left(T_{w}-T_{\infty}\right) x^{* 3} / v^{2} \\
& f(\xi, \eta)=\frac{\psi}{r v\left(G r_{x}\right)^{1 / 4}}, \quad N(\xi, \eta)=\frac{v\left(G r_{x} \cdot\right)^{3 / 4}}{\left(x^{*}\right)^{2}} g(\xi, \eta), \theta(\xi, \eta)=\frac{T-T_{\infty}}{T_{w}-T_{\infty}}, \\
& \phi(\xi, \eta)=\frac{c-c_{\infty}}{c_{w}-c_{\infty}} \\
& u=\frac{v\left(G r_{x} \cdot\right)^{1 / 2}}{x^{*}} f^{\prime}=U_{n} f^{\prime}, v=-\frac{v\left(G r_{x} \cdot\right)^{1 / 4}}{x^{*}}\left[\left(\frac{\xi}{1+\xi}+\frac{3+n}{4}\right) f+\xi \frac{\partial f}{\partial \xi}+\frac{n-1}{4} \eta f^{\prime}\right],
\end{aligned}
$$

where $U_{r}$ is a reference velocity.

Substituting Equations (9) and (10) into Equations (1) through (7) yields the following non-similar dimensionless equations:

$$
\begin{aligned}
& (1+K) f^{\prime \prime \prime}+K g^{\prime}+\left(\frac{\xi}{1+\xi}+\frac{3+n}{4}\right) f f^{\prime \prime}-\frac{1+n}{2}\left(f^{\prime}\right)^{2}-H a^{2} \xi^{1 / 2} f^{\prime}+\theta+\Lambda \phi=\xi\left(f^{\prime} \frac{\partial f^{\prime}}{\partial \xi}-f^{\prime \prime} \frac{\partial f}{\partial \xi}\right) \\
& \left(1+\frac{K}{2}\right) g^{\prime \prime}+\left(\frac{\xi}{1+\xi}+\frac{3+n}{4}\right) f g^{\prime}-\frac{3 n+1}{4} f^{\prime} g-K B \xi^{1 / 2}\left(2 g+f^{\prime \prime}\right)=\xi\left(f^{\prime} \frac{\partial g}{\partial \xi}-g^{\prime} \frac{\partial f}{\partial \xi}\right), \\
& \frac{\theta^{\prime \prime}}{\operatorname{Pr}}+\left(\frac{\xi}{1+\xi}+\frac{3+n}{4}\right) f \theta^{\prime}-n f^{\prime} \theta+\frac{4}{3} \frac{R_{d}}{\operatorname{Pr}}\left\{\theta^{\prime}\left[\left(r_{t}-1\right) \theta+1\right]^{3}\right\}^{\prime}=\xi\left(f^{\prime} \frac{\partial \theta}{\partial \xi}-\theta^{\prime} \frac{\partial f}{\partial \xi}\right), \\
& \frac{\phi^{\prime \prime}}{S c}+\left(\frac{\xi}{1+\xi}+\frac{3+n}{4}\right) f \phi^{\prime}-n f^{\prime} \phi-\gamma \xi^{1 / 2} \phi=\xi\left(f^{\prime} \frac{\partial \phi}{\partial \xi}-\phi^{\prime} \frac{\partial f}{\partial \xi}\right), \\
& \eta=0: f^{\prime}=0, \quad g=-n f^{\prime \prime}(\xi, 0), f_{o} \xi^{1 / 4}=\left(\frac{\xi}{1+\xi}+\frac{3+n}{4}\right) f+\xi \frac{\partial f}{\partial \xi}, \quad \theta=1, \quad \phi=1 \text {, } \\
& \eta \rightarrow \infty: f^{\prime} \rightarrow 0, \quad g \rightarrow 0, \theta \rightarrow 0, \quad \phi \rightarrow 0,
\end{aligned}
$$

where a prime denotes partial differentiation with respect to $\eta$ and

$$
\begin{aligned}
& H a^{2}=\frac{\sigma B_{o}^{2}}{\rho v}\left(\frac{x_{o}^{4}}{G r_{x_{o}}}\right)^{1 / 2}, \Lambda=\frac{\beta_{c}\left(c_{w}-c_{\infty}\right)}{\beta_{T}\left(T_{w}-T_{\infty}\right)}, \operatorname{Pr}=\frac{v}{\alpha}, R_{d}=\frac{4 \sigma T_{\infty}^{3}}{\left[k\left(a_{r}+\sigma_{s}\right)\right]}, B=\frac{x_{o}^{2}}{j}\left(G r_{x_{o}}\right)^{-1 / 2}, r_{t}=\frac{T_{w}}{T_{\infty}} \\
& K=\frac{k}{\mu}, S c=\frac{v}{D}, \quad \gamma=k_{r} x_{0}^{2} / v\left(G r_{x_{0}}\right)^{1 / 2}, f_{o}=\frac{v_{o}}{v}\left(\frac{x_{o}^{4}}{G r_{x_{o}}}\right)^{1 / 4}
\end{aligned}
$$

are the square of the Hartmann number, concentration to thermal buoyancy ratio, Prandtl number, radiationconduction parameter, spin gradient viscosity parameter, micropolar fluid vortex viscosity parameter, surface temperature parameter, Schmidt number, dimensionless chemical reaction parameter and mass transfer Coupled heat and mass transfer by MHD natural convection of micropolar fluid about a truncated cone in the presence of... 
coefficient, respectively. It is should be noted that positive values of $f_{0}$ indicate fluid suction at the cone surface while negative values of $f_{0}$ indicate fluid blowing or injection at the wall.

Equations (9) through (11) represent general equations which include various special cases. For example, by formally setting all of $\mathrm{Ha}, \mathrm{N}$ and $n$ equal to zero, Equations (9) and (10) reduce to those reported earlier by Yih (1999) in his work concerning laminar natural convection over a truncated cone with uniform wall temperature and concentration the absence of a magnetic field and mass diffusion effect. Also, in the absence of chemical reaction $(\gamma=0)$, for constant wall temperature and concentration $(n=0)$, Equations (9) and (10) reduce to those reported by Chamkha (2001).

The local skin-friction coefficient $C_{f}$, local Nusselt number $N u_{x^{*}}$, local wall couple stress $M_{w}$, and the local Sherwood number $S h_{x^{*}}$ are important physical properties. These can be defined in dimensionless form below as given by Yih (1999) and Chamkha (2001).

$$
\begin{aligned}
& C_{f}=-2\left(G r_{x^{*}}\right)^{-1 / 4} f^{\prime \prime}(\xi, 0), \quad N u_{x^{*}}=-\left(1+\frac{4 R_{d} r_{t}^{3}}{3}\right)\left(G r_{x^{*}}\right)^{1 / 4} \theta^{\prime}(\xi, 0) \\
& M_{w}=\frac{\gamma^{*} \mu\left(G r_{x^{*}}\right)^{-1 / 4}}{x^{\bullet^{3}}} g^{\prime}(\xi, 0), S h_{x^{*}}=-\left(G r_{x^{*}}\right)^{-1 / 4} \phi^{\prime}(\xi, 0) .
\end{aligned}
$$

\section{Numerical Method}

The initial-value problem represented by Equations (11) through (16) with $\xi$ playing the role of time is nonlinear and has no closed-form solution. Therefore, it must be solved numerically. The implicit, tri-diagonal, finitedifference method discussed by Blottner (1970) has proven to be adequate for the solution of boundary-layer equations accurately. For this reason, it is adopted in this work. All first-order derivatives with respect to $\xi$ are replaced by two-point backward difference quotients such that; $\frac{\partial A}{\partial \xi}=\frac{A_{m, n}-A_{m-1, n}}{\Delta \xi_{m-1}}$,

where $A$ is a typical independent variable; $m$ and $n$ indicate lines of constant $\xi$ and constant $\eta$, respectively. $\Delta \xi_{m-}$ ${ }_{1}$ is the $\xi$ step size between the $m-1$ and $m$ lines of constant Equation (9) is converted into a second-order partial differential equation by letting $V=f^{\prime}$. Then, all equations governing $V, g, \theta$ and $\phi$ can be written in the general form

$$
\pi_{1} Z^{\prime \prime}+\pi_{2} Z^{\prime}+\pi_{3} Z+\pi_{4}=0
$$

where $Z=V, g, \theta$, or $\phi$ and the $\pi$ 's are constants, functions of the dependent variables, or functions of the independent variables. These equations are discretized using three-point central-difference quotients and, as a consequence, a set of algebraic equations results at each line of constant $\xi$. These algebraic equations are then solved by the well-known Thomas algorithm (see Blottner (1970)) with iteration to deal with the non-linearities of the problem. When the solution at a specific line of constant $\xi$ is obtained, the same solution procedure is used for the next line of constant $\xi$. This marching process continues until the desired value of $\xi$ is reached. At each line of constant $\xi$, when $V$ is known, the equation $f^{\prime}=V$ is then solved for $f$ using the trapezoidal rule. The convergence criterion employed was based on the difference between the current and the previous iterations. When this difference reached $10^{-5}$, the solution was assumed converged and the iteration procedure was terminated. Variable step sizes in the $\eta$ direction and constant step sizes in the $\xi$ direction were utilized in order to accommodate the sharp changes in the dependent variables especially in the immediate vicinity of the cone surface. The $(\xi, \eta)$ computational domain consisted of 101 and 196 points, respectively. The constant step size in $\xi$ was taken to be $10^{-2}$ while the initial step size in $\eta$ was taken to be equal to $10^{-3}$ and the growth factor was taken to be 1.04 . This gave $\eta_{\infty}=150$. These values were found to give accurate grid-independent results as verified by the comparisons mentioned below. In order to access the accuracy of the numerical results, various comparisons with the previously published work of Yih (1999) and Chamkha (2001) for the cases of a vertical plate $(\xi=0)$ and a full cone $(\xi=\infty)$ were performed. These comparisons are presented in Tables 1 and 2 . It is obvious from these tables that excellent agreement between the results exist. These favourable comparisons lend confidence in the graphical results to be reported in the next section. 
Table 1: Comparison of values of $f^{\prime \prime}(0,0)$ and $-\theta^{\prime}(0,0)$ for various values of $\operatorname{Pr}$ with $f_{o}=0, n=0, H a=\Lambda=0, N=0$, $\gamma=0$ and $R_{d}=0$.

\begin{tabular}{|c|c|c|c|c|c|c|}
\hline \multirow{2}{*}{$P r$} & \multicolumn{3}{|c|}{$f^{\prime \prime}(0,0)$} & \multicolumn{3}{|c|}{$\theta^{\prime}(0,0)$} \\
\cline { 2 - 6 } & Yih (1999) & $\begin{array}{c}\text { Chamkha } \\
(2001)\end{array}$ & $\begin{array}{c}\text { Present } \\
\text { results }\end{array}$ & Yih (1999) & $\begin{array}{c}\text { Chamkha } \\
(2001)\end{array}$ & $\begin{array}{c}\text { Present } \\
\text { results }\end{array}$ \\
\hline 0.0001 & 1.4998 & 1.4997 & 1.4997 & 0.0060 & 0.0059 & 0.0059 \\
0.001 & 1.4728 & 1.4727 & 1.4727 & 0.0189 & 0.0188 & 0.0188 \\
0.01 & 1.3968 & 1.3965 & 1.3965 & 0.0570 & 0.0574 & 0.0574 \\
0.1 & 1.2144 & 1.2151 & 1.2151 & 0.1629 & 0.1630 & 0.1630 \\
1 & 0.9084 & 0.9081 & 0.9081 & 0.4012 & 0.4015 & 0.4015 \\
10 & 0.5927 & 0.5927 & 0.5927 & 0.8266 & 0.8274 & 0.8274 \\
100 & 0.3559 & 0.3558 & 0.3558 & 1.5493 & 1.5503 & 1.5503 \\
1000 & 0.2049 & 0.2049 & 0.2049 & 2.8035 & 2.8044 & 2.8044 \\
10,000 & 0.1161 & 0.1161 & 0.1161 & 5.0127 & 5.0131 & 5.0131 \\
\hline
\end{tabular}

Table 2: Comparison of values of $f^{\prime \prime}(\infty, 0)$ and $-\theta^{\prime}(\infty, 0)$ for various values of $\operatorname{Pr}$ with $f_{\mathrm{o}}=0, n=0, \mathrm{Ha}=\Lambda=0, N=$ $0, \gamma=0$ and $R_{d}=0$.

\begin{tabular}{|c|c|c|c|c|c|c|}
\hline \multirow{2}{*}{$P r$} & \multicolumn{3}{|c|}{$\mathrm{f}^{\prime \prime}(\infty, 0)$} & \multicolumn{3}{c|}{$\theta^{\prime}(\infty, 0)$} \\
\cline { 2 - 6 } & Yih (1999) & $\begin{array}{c}\text { Chamkha } \\
(2001)\end{array}$ & $\begin{array}{c}\text { Present } \\
\text { results }\end{array}$ & Yih (1999) & $\begin{array}{c}\text { Chamkha } \\
(2001)\end{array}$ & $\begin{array}{c}\text { Present } \\
\text { results }\end{array}$ \\
\hline 0.0001 & 1.6006 & 1.6005 & 1.6005 & 0.0079 & 0.0078 & 0.0078 \\
0.001 & 1.5135 & 1.5133 & 1.5133 & 0.0246 & 0.0245 & 0.0245 \\
0.01 & 1.3551 & 1.3549 & 1.3549 & 0.0749 & 0.0751 & 0.0751 \\
0.1 & 1.0960 & 1.0962 & 1.0962 & 0.2116 & 0.2116 & 0.2116 \\
1 & 0.7699 & 0.7697 & 0.7697 & 0.5109 & 0.5111 & 0.5111 \\
10 & 0.4877 & 0.4877 & 0.4877 & 1.0339 & 1.0342 & 1.0342 \\
100 & 0.2896 & 0.2895 & 0.2895 & 1.9226 & 1.9230 & 1.9230 \\
1000 & 0.1661 & 0.1661 & 0.1661 & 3.4696 & 3.4700 & 3.4700 \\
10,000 & 0.0940 & 0.0940 & 0.0940 & 6.1984 & 6.1988 & 6.1988 \\
\hline
\end{tabular}

\section{Results and Discussion}

In this section, a detailed parametric study has been performed for the effects of the Hartmann number $H a$, micropolar vortex viscosity parameter $K$, wall temperature and concentration exponent $\mathrm{n}$, thermal radiation parameter $R_{d}$, dimensionless chemical reaction parameter $\gamma$, and the suction/injection parameter $f_{o}$ on the velocity, microrotation, temperature, and concentration profiles as well as the local skin-friction coefficient, local wall couple stress, local Nusselt number and the local Sherwood number is presented graphically in Figures 2 through 19. All data are provided in the legends of these figures correspond to a micropolar fluid having a high vortex viscosity $(K=2)$ i.e. is strongly non-Newtonian (unless otherwise indicated) with $B=1.0$. Since $\Lambda=1$ in these figures, the thermal and species buoyancy forces are of the same order of magnitude and assist each other. $\operatorname{Pr}$ is set as 7.0 corresponding to water and $S c=0.78$ implies a dominance of momentum diffusivity over species diffusivity.

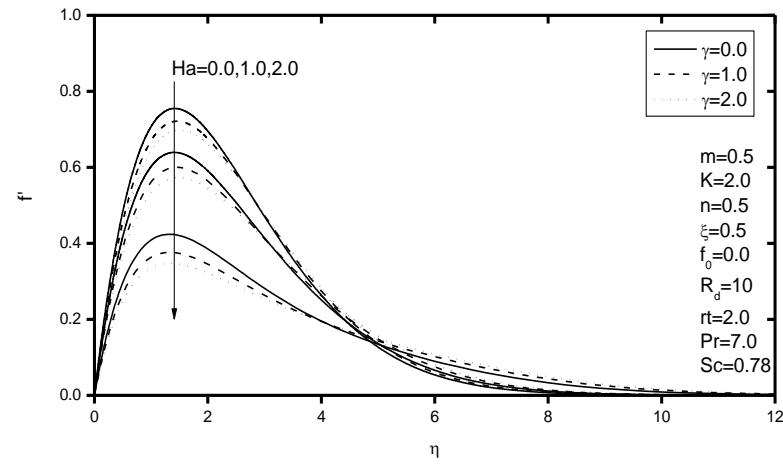

Figure 2: Effects of $\mathrm{Ha}$ and $\gamma$ on velocity profiles

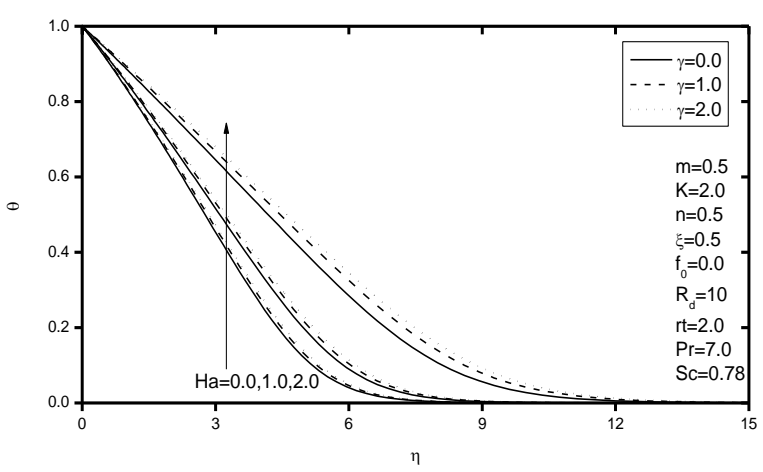

Figure 4: Effects of $\mathrm{Ha}$ and $\gamma$ on temperature profiles 


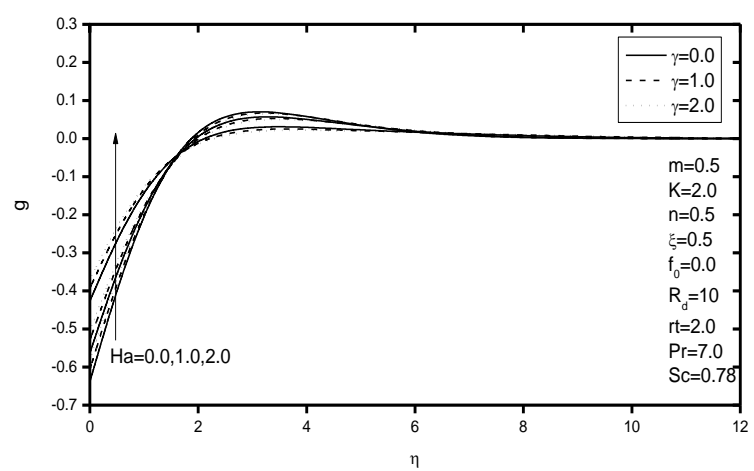

Figure 3: Effects of $H a$ and $\gamma$ on microrotation profiles

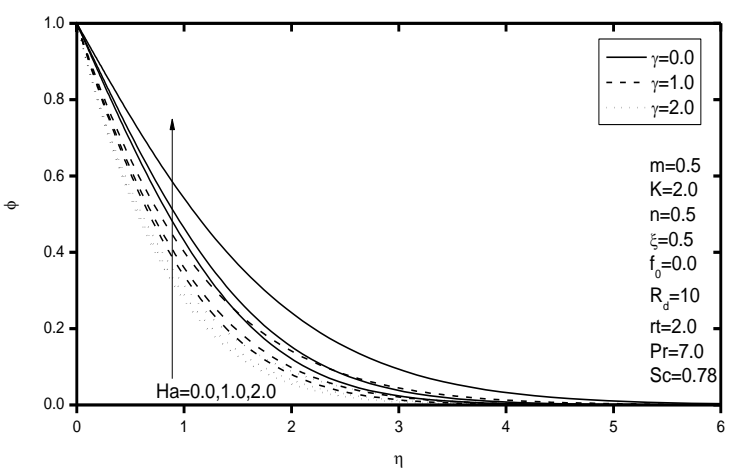

Figure 5: Effects of $\mathrm{Ha}$ and $\gamma$ on concentration profiles

Figures 2-5 show the effects of the Hartmann number $\mathrm{Ha}$ and the chemical reaction parameter $\gamma$ on the fluid velocity $f^{\prime}$, angular velocity field (microrotation) $g$, temperature $\theta$ and concentration $\phi$ profiles, respectively. The results show that the presence of the magnetic force causes retardation of the fluid motion represented by general decreases in the fluid velocity and angular velocity (except far away the wall which is governed by the special boundary condition there) and increases in its temperature and solute concentration. These behaviors in $f^{\prime}, \theta$, and $\phi$ are accompanied by increases in all of the hydrodynamic, thermal, and concentration boundary layers as $\mathrm{Ha}$ increases. On other hand, it is seen that the velocity, angular velocity (microrotation) and concentration profiles decrease with increases in the chemical reaction parameter, while a small increase in the temperature profiles occurs. This shows that the diffusion rates can be tremendously altered by chemical reactions. It is also important to note that increasing the chemical reaction parameter significantly alters the concentration boundary layer thickness without any significant effect on the momentum and thermal boundary layers.

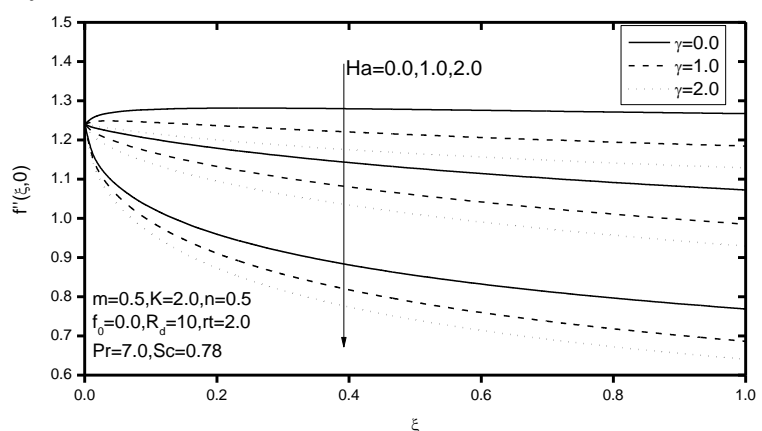

Figure 6: Effects of $\mathrm{Ha}$ and $\gamma$ on development of skinfriction coefficient

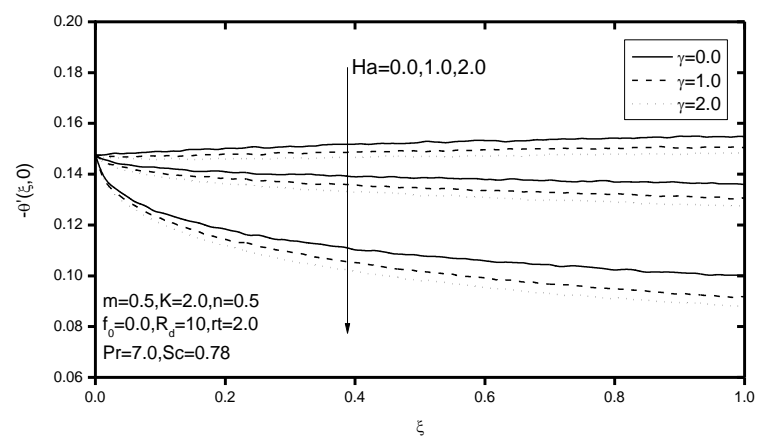

Figure 8: Effects of $\mathrm{Ha}$ and $\gamma$ on development of Nusselt number

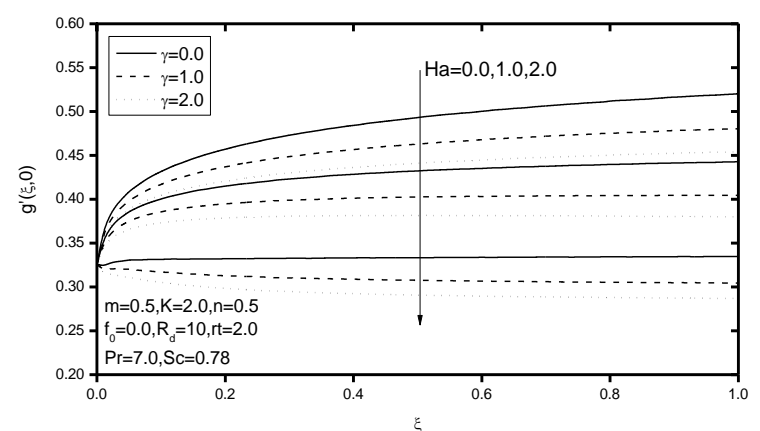

Figure 7: Effects of $\mathrm{Ha}$ and $\gamma$ on development of wall couple stress

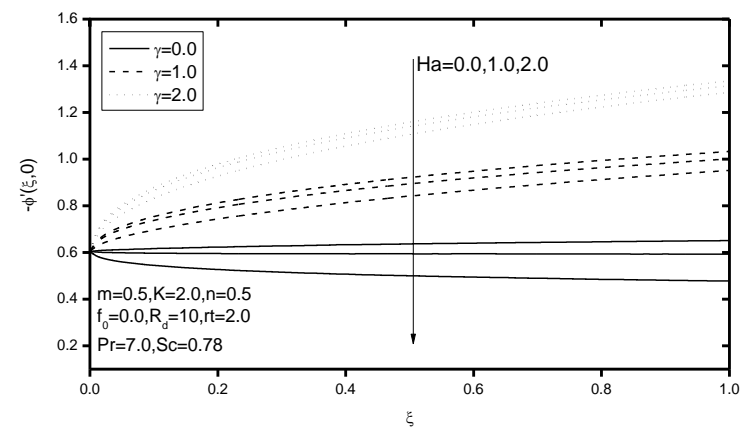

Figure 9: Effects of $\mathrm{Ha}$ and $\gamma$ on development of Sherwood number

Figures 6-9 present the effects of the Hartmann number $H a$ on the local skin-friction coefficient $C_{f}$, local wall couple stress $M_{w}$, local Nusselt number $N u_{x}$, and the local Sherwood number $S h_{x}$, respectively. As seen from the definitions of $C_{f}, M_{w}, N u_{x}$, and $S h_{x}$, they are directly proportional to $f^{\prime \prime}(\xi, 0), g^{\prime}(\xi, 0),-\theta^{\prime}(\xi, 0)$ and $-\phi^{\prime}(\xi, 0)$, respectively. For this reason, these are shown in Figures 6-9. It was observed from Figures 2-5 that the wall 
slope of the velocity and microrotation profiles decreases while the slopes of the temperature and concentration profiles increase as $\mathrm{Ha}$ increases. This produces reductions in all of $C_{f}, M_{w}, N u_{x}$, and $S h_{x}$ as $H a$ increases as depicted in Figures 6 through 9. It is also observed from these figures that in the presence of magnetic effect at $H a=1.0$ and 2.0, all of $f^{\prime \prime}(\xi, 0),-\theta^{\prime}(\xi, 0)$ and $-\phi^{\prime}(\xi, 0)$ decrease with increasing values of $\xi$ while $g^{\prime}(\xi, 0)$ increases and the opposite behavior occurs in the absence of magnetic effect for $H a=0$. Further, it can be seen that as $\gamma$ increases, the Sherwood number increases while the opposite effect is found for the skin-friction coefficient, wall couple stress and the Nusselt number. This is because as $\gamma$ increases, the concentration difference between the cone surface and the fluid decreases and so the rate of mass transfer at the wall must increase while both of the skin-friction coefficient and wall couple stress decrease as a result of the decrease in the flow and the microrotation velocities of the fluid.

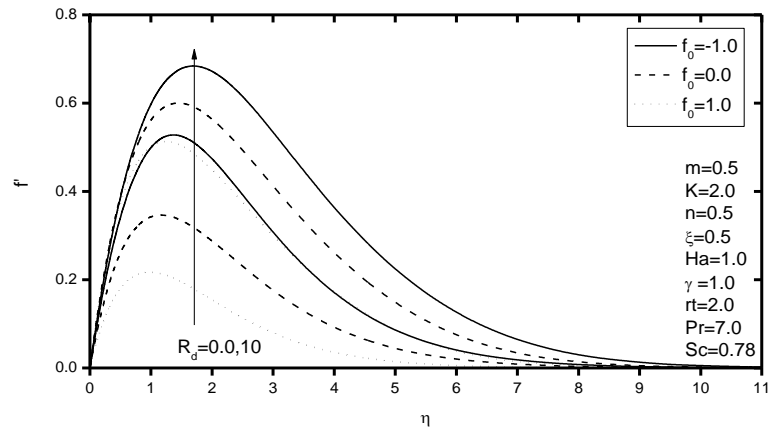

Figure 10: Effects of $f_{o}$ and $R_{d}$ on velocity profiles

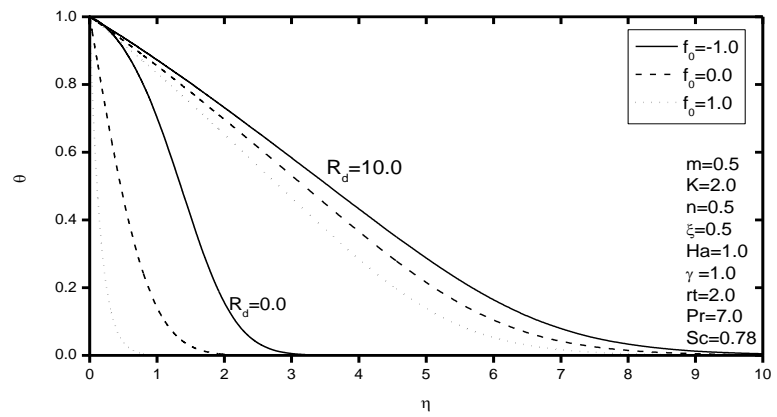

Figure 12: Effects of $f_{o}$ and $R_{d}$ on temperature profiles

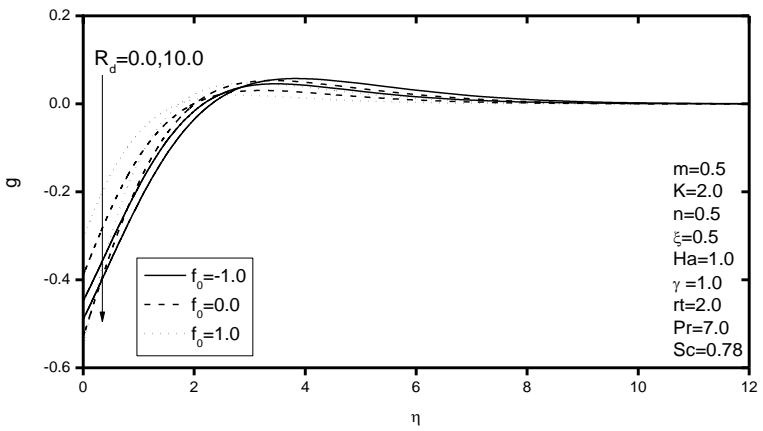

Figure 11: Effects of $f_{o}$ and $R_{d}$ on microrotation profiles

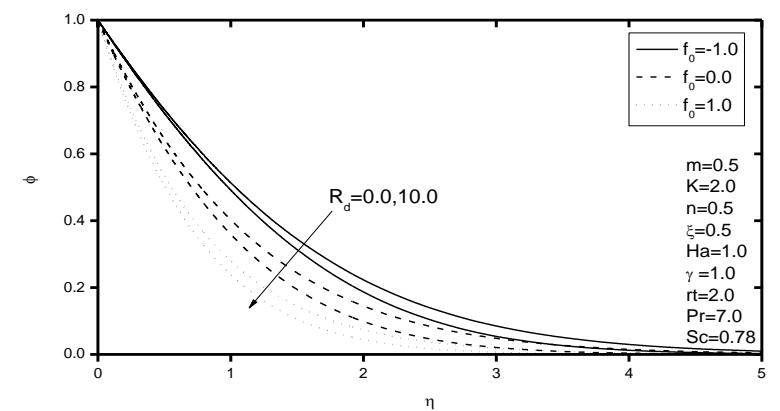

Figure 13: Effects of $f_{o}$ and $R_{d}$ on concentration profiles

Figures 10-13 illustrate the velocity, angular velocity (microrotation), temperature and concentration profiles with variation in the wall suction/blowing parameter $f_{o}$ and the radiation-conduction parameter $R_{d}$, respectively. Imposition of fluid suction $\left(f_{o}>0\right)$ at the cone surface has a tendency to reduce all of the hydrodynamic, thermal and concentration boundary layers. This causes all of the velocity, temperature and concentration to decrease, whereas the micro-rotation increases with negative values at every point far from the surface. On the other hand, injection of fluid $\left(f_{o}<0\right)$ through the cone surface produces the opposite effect, namely increases in all of the velocity, temperature and concentration and decrease in the micro-rotation profiles. These behaviours are clearly shown in Figures 10 through 13. Consistent with the behaviour reported by Yih (1999) and Chamkha (2001), increasing the value of $R_{d}$ results in increases in both the velocity and temperature profilers and the maximum velocity tends to move away from the cone surface, whereas the decrease. However, both of the angular velocity (microrotation) and concentration profiles tend to decrease as a result of increasing the radiation effect as observed from Figures 10 and 13.

The effects of both $f_{o}$ and $R_{d}$ on the development of the local skin-friction coefficient [or $\left.f^{\prime \prime}(\xi, 0)\right]$, the local wall couple stress [or $g^{\prime}(\xi, 0)$ ], the local Nusselt number [or - $\left.\theta^{\prime}(\xi, 0)\right]$ and the local Sherwood number [or $\left.-\phi^{\prime}(\xi, 0)\right]$ are displayed in Figures 14 through 17, respectively. It is predicted that as the wall suction/blowing parameter $f_{o}$ increases, the local skin-friction coefficient decreases (only in the absence of radiation effect) while the local wall couple stress coefficient, local Nusselt number and the local Sherwood number increase. In addition, it is observed from these figures that increasing the value of the radiation parameter $R_{d}$ causes increases in the local skin friction coefficient, local wall couple stress, and the local Sherwood number. However, the local Nusselt number tends to decrease due to increases in the values of the radiation parameter $R_{d}$. 


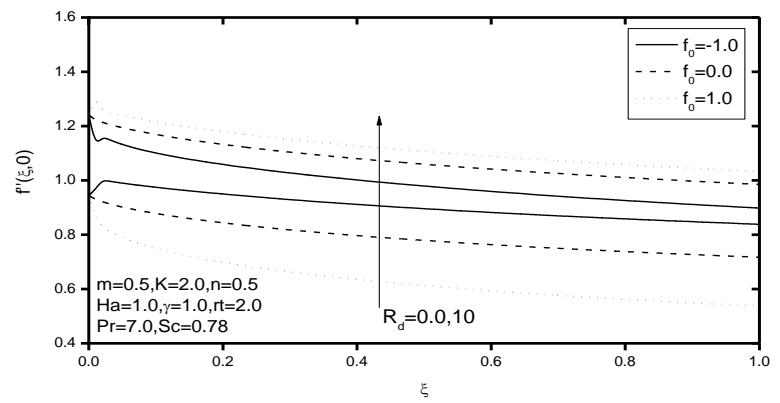

Figure 14: Effects of $f_{o}$ and $R_{d}$ on development of skinfriction coefficient

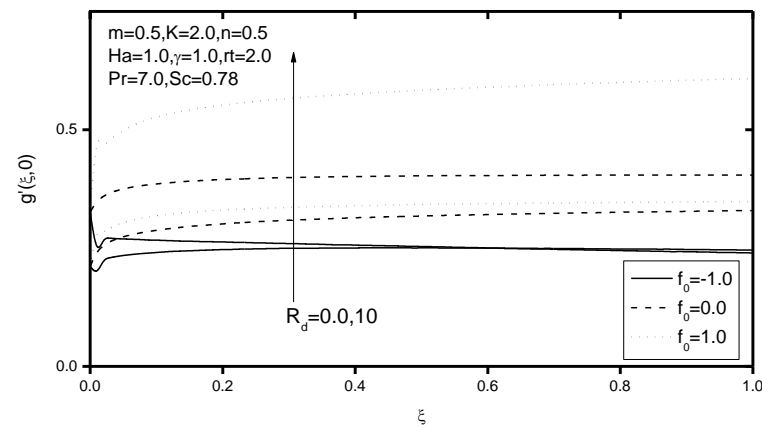

Figure 15: Effects of $f_{o}$ and $R_{d}$ on development of wall couple stress

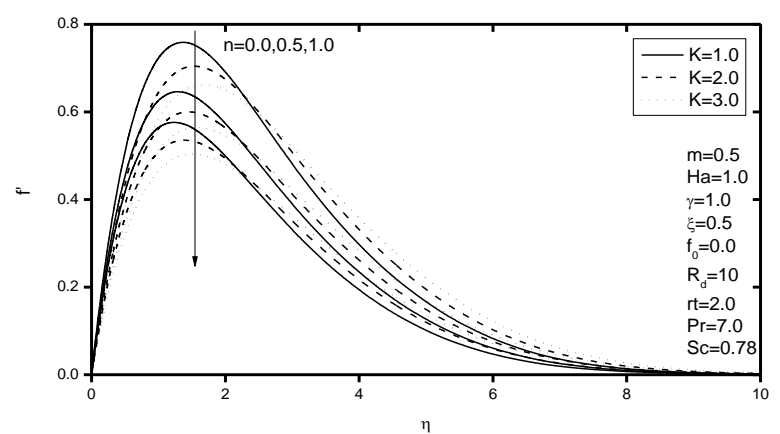

Figure 18: Effects of $K$ and $n$ on velocity profiles

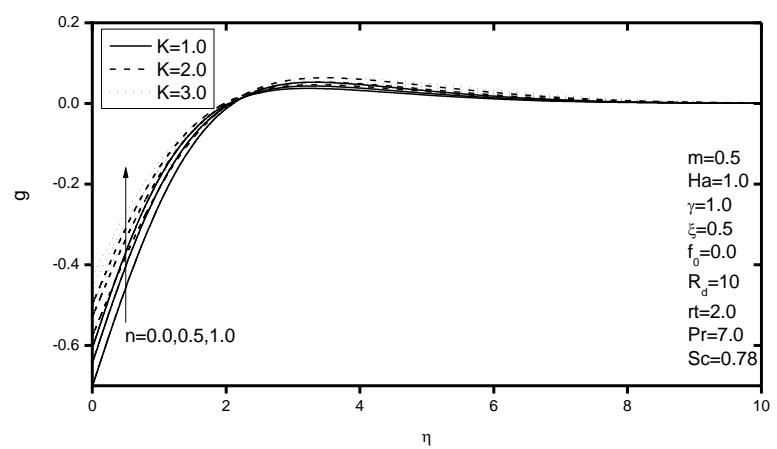

Figure 20: Effects of $K$ and $n$ on microrotation profiles

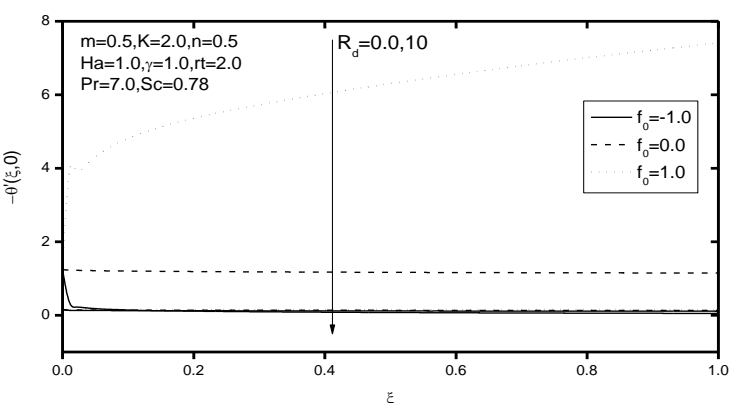

Figure 16: Effects of $f_{o}$ and $R_{d}$ on development of Nusselt number

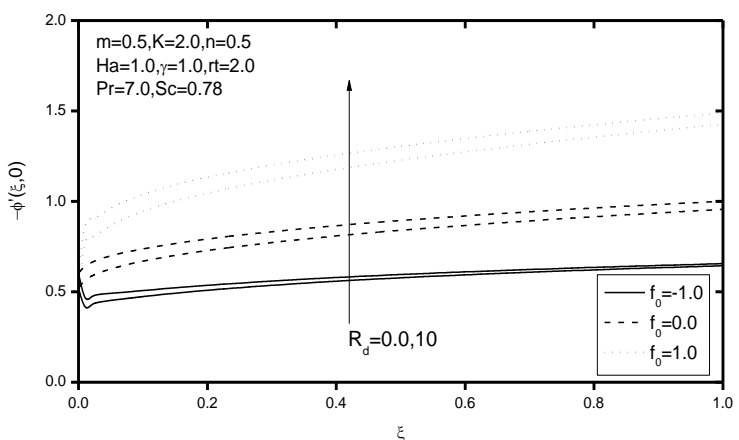

Figure 17: Effects of $f_{o}$ and $R_{d}$ on Development of Sherwood number

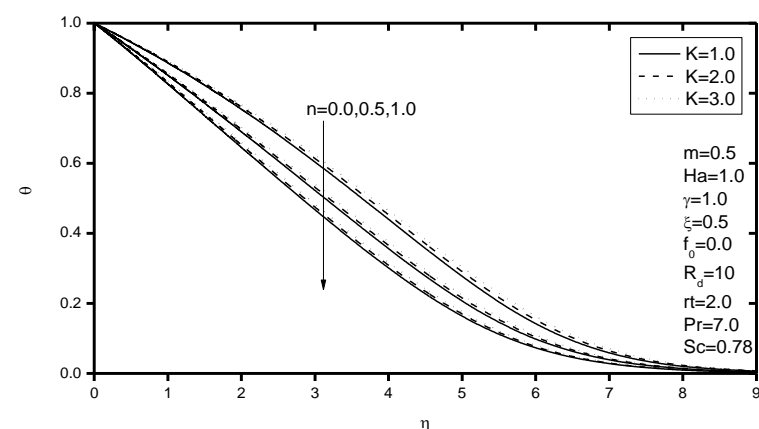

Figure 19: Effects of $K$ and $n$ on temperature profiles

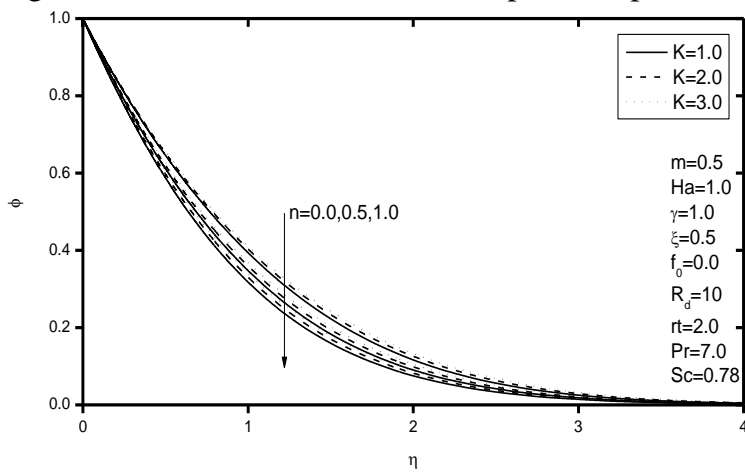

Figure 21: Effects of $K$ and $n$ on concentration profiles

In Figures 18-21, the effects of the wall temperature and concentration exponent $n$ and the micropolar fluid vortex viscosity parameter $K$ on the velocity, temperature, and concentration profiles are respectively presented. For a given value of $K$, an increase in the exponent $n$ tends to decelerate the flow around the cone surface and the angular velocity with reductions in the temperature and concentration profiles as depicted in Figures 18 
through 21. Furthermore, it is observed from these figures that for a given value of $n$, as the vortex viscosity parameter $K$ increases, the angular velocity and the concentration profiles increase whereas both of the velocity and temperature profiles decrease.

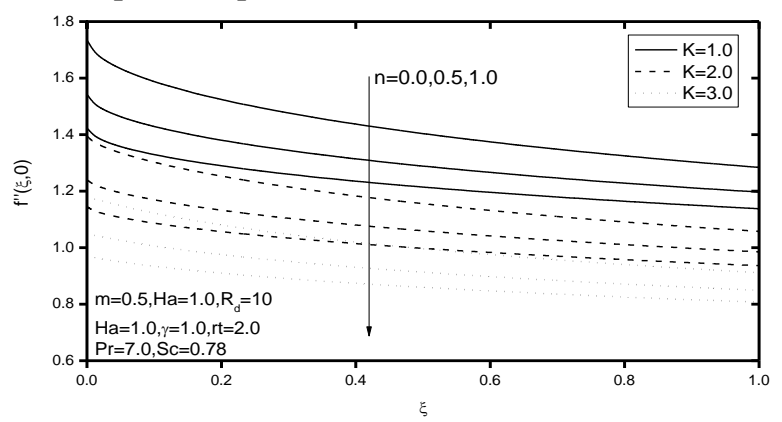

Figure 22: Effects of $K$ and $n$ on development of skinfriction coefficient

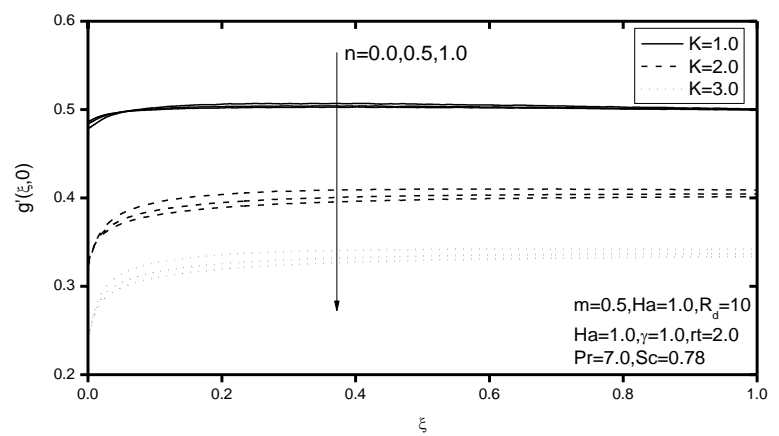

Figure 23: Effects of $K$ and $n$ on development of wall couple stress

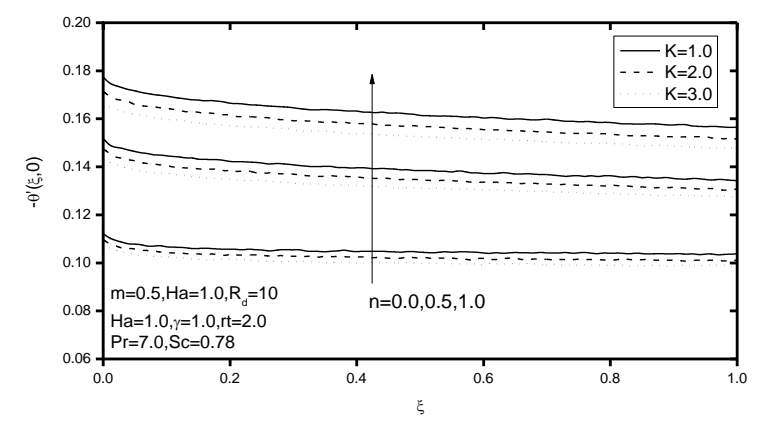

Figure 24: Effects of $K$ and $n$ on development of Nusselt number

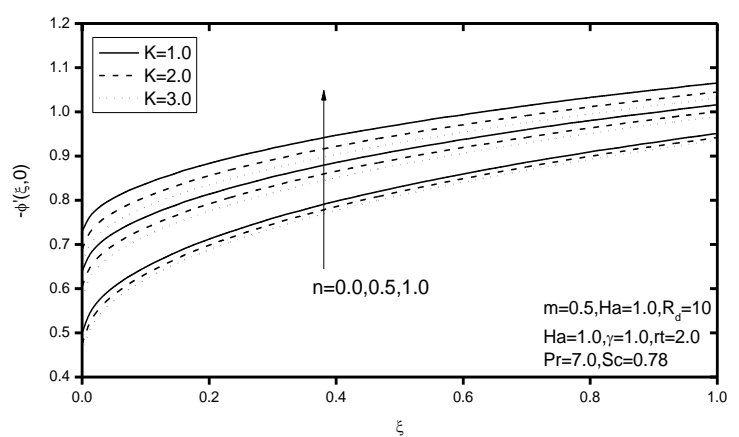

Figure 25: Effects of $K$ and $n$ on Development of Sherwood number

In Figures 22 through 25, the effects of $n$ and $K$ on the values of $C_{f}, M_{w}, N u_{x}$, and $S h_{x}$. are presented, respectively. In these figures, it is predicted that, regardless of the value of $K$, an increase in the value of $n$ results in a decrease in both of the local friction coefficient $\mathrm{C}_{\mathrm{f}}$ and the local wall couple stress $M_{w}$ while higher heat transfer rates $N u_{x}$. can be obtained by increasing the value of $n$ for certain values of $\xi$. Moreover, it is seen that the mass transfer is enhanced by increasing the value of $n$. In addition, as the vortex viscosity parameter $K$ increases, all values of $C_{f}, M_{w}, N u_{x}$, and $S h_{x}$. decrease. It should also be noted that the increase in the local wall couple stress due to decreasing the value of the viscosity parameter $K$ is more pronounced than for the local friction coefficient, local Nusselt and Sherwood numbers.

\section{Conclusion}

In this paper, the effects of chemical reaction and thermal radiation on the coupled heat and mass transfer by MHD natural convection boundary-layer flow of a micropolar fluid over a permeable truncated cone with variable surface temperature and concentration were studied. The governing boundary-layer equations were transformed into a non-similar form, and these equations were solved numerically. The effects of magnetic field, thermal radiation, chemical reaction, injection or suction and power-law variations of both the wall temperature and concentration on the velocity, microrotation, temperature and concentration profiles as well as the local skin-friction coefficient, local couple stress, local Nusselt number and the local Sherwood number were shown graphically and discussed. Increasing the magnetic field parameter and the micropolar fluid vortex viscosity parameter resulted in decreases in all of the local skin-friction coefficient, local couple stress, local Nusselt and Sherwood numbers. The presence of a homogeneous chemical reaction led to decreases in the local skin-friction coefficient, local wall couple stress and the local Nusselt number while the local Sherwood number increased. Increasing the suction/injection parameter resulted in reduction in the local skin-friction coefficient whereas the local couple stress, local Nusselt and Sherwood numbers local couple stress, local Nusselt and Sherwood numbers enhanced. In addition, the presence of a thermal radiation led to increases in the local skin-friction coefficient, local couple stress, local Sherwood number while the local Nusselt number decreased. Increasing the wall temperature and concentration exponent reduced both the local skin-friction coefficient and the local couple stress whereas both the local Nusselt and Sherwood numbers increased 


\section{References}

Ariman, T., Turk, M.A. and Sylvester, N.D. (1973): Microcontinuum fluid mechanics-a review, International Journal of Engineering Science, Vol. 11, No. 8, pp. 905-930. http://dx.doi.org/10.1016/0020-7225(73)90038-4 Ariman, T., Turk, M.A. and Sylvester, N.D., (1974): Applications of microcontinuum fluid mechanics, International Journal of Engineering Science, Vol. 12, No. 4, pp. 273-293. http://dx.doi.org/10.1016/0020$\underline{7225(74) 90059-7}$

Blottner, F.G. (1970): Finite-difference methods of solution of the boundary-layer equations, AIAA Journal, Vol. 8, No. 2, pp. 193-205. http://dx.doi.org/10.2514/3.5642

Chamkha, A.J. (2001): Coupled heat and mass transfer by natural convection about a truncated cone in the presence of magnetic field and radiation effects, Numerical Heat Transfer, Part A, Vol. 39, No. 5, pp. 511-530. http://dx.doi.org/ 10.1080/104077801750111601

Chamkha, A.J., Mohamed, R.A. and Ahmed, S.E. (2011): Unsteady MHD natural convection from a heated vertical porous plate in a micropolar fluid with Joule heating, chemical reaction and radiation effects, Meccanica, vol. 46, No. 2, pp. 399-411. http://dx.doi.org/10.1007/s11012-010-9321-0

Chang, C.-L. (2008): Numerical simulation for natural convection of micropolar fluids flow along slender hollow circular cylinder with wall conduction effect, Communications in Nonlinear Science and Numerical Simulation, Vol. 13, No. 3, pp. 624-636. http://dx.doi.org/10.1016/j.cnsns.2006.05.012

Cheng, C.Y. (2008): Natural convection of a micropolar fluid from a vertical truncated cone with power-law variation in surface temperature, International Communications in Heat and Mass Transfer, Vol. 35, No. 1, pp. 39-46. http://dx.doi.org/10.1016/j.icheatmasstransfer.2007.05.018

Damseh, R.A., Al-Odat, M.Q., Chamkha, A.J. and Shannak, B.A. (2009): Combined effect of heat generation or absorption and first-order chemical reaction on micropolar fluid flows over a uniformly stretched permeable surface, Int. J. Thermal Sciences, Vol. 48, No. 8, pp. 1658-1663. http://dx.doi.org/10.1016/j.ijthermalsci.2008.12.018

EL-Kabeir, S.M.M. (2004): Radiative effects on forced convection flows in micropolar fluids with variable viscosity, Can. J. Phys., Vol. 82, No. 2, (2004) pp. 151-165. http://dx.doi.org/10.1139/p03-085

EL-Kabeir, S.M.M. (2005): Hiemenz flow of a micropolar viscoelastic fluid in hydromagnetics, Can. J. Phys., Vol. 83, No. 10, pp. 1007-1017. http://dx.doi.org/10.1139/p05-039

EL-Kabeir, S.M.M. and Modather, M. (2007): Chemical reaction, heat and mass transfer on MHD flow over a vertical isothermal cone surface in micropolar fluids with heat generation/absorption, Applied Mathematical Sciences, Vol. 1, No. 34, pp. 1663 -1674.

EL-Kabeir, S.M.M., Chamkha, A.J. and Rashad, A.M. (2010): Heat and mass transfer by MHD stagnation-point flow of a power-law fluid towards a stretching surface with radiation, chemical reaction and Soret And Dufour effects, International Journal of Chemical Reactor Engineering, Vol. 8, No. 1, pp. 1-18. http://dx.doi.org/10.2202/1542-6580.2396

EL-Kabeir, S.M.M., Rashad, A.M. and Gorla, R.S.R. (2011): Heat Transfer in a micropolar fluid flow past a permeable continuous moving surface, ZAMM, Vol. 91, Issue 5, (2011) 360-370. http://dx.doi.org/10.1002/zamm.200800049

Erigen, A.C. (1966): Theory of micropolar fluids, Journal of Mathematics and Mechanics, Vol. 16, pp. 1-18.

Erigen, A.C. (1972): Theory of the momicropolar fluids, Journal of Mathematical Analysis and Application, Vol. 38, pp. 480-496.

Kishan, N. and Amrutha, P. (2011): Effects of viscous dissipation on MHD flow with heat and mass transfer over a stretching surface with heat source, thermal stratification and chemical reaction, Journal of Naval Architecture and Marine Engineering, Vol. 7, No. 1, pp. 11-18. http://dx.doi.org/ 10.3329/jname.v7i1.3254

Modather, M., Rashad, A.M. and Chamkha, A.J. (2009): An analytical study On MHD heat and mass transfer oscillatory flow of micropolar fluid over a vertical permeable plate in a porous medium, Turkish J. Eng. Env. Sci., Vol. 33, pp. 245-257.

Pal, D. (2011): Combined effects of non-uniform heat source/sink and thermal radiation on heat transfer over an unsteady stretching permeable surface, Commun Nonlinear Sci Numer Simulat., Vol. 16, No. 4, pp. 1890-1904. http://dx.doi.org/10.1016/j.cnsns.2010.08.023

Pal, D., and Chatterjee, S. (2010): Heat and mass transfer in MHD non-Darcian flow of a micropolar fluid over a stretching sheet embedded in a porous media with non-uniform heat source and thermal radiation, Commun. Nonlinear Sci. Numer. Simulat., Vol. 15, No. 7, pp. 1843-1857. http://dx.doi.org/10.1016/j.cnsns.2009.07.024 Rashad, A.M. (2013): Effects of radiation and variable viscosity on unsteady MHD flow of a rotating fluid from stretching surface in porous Medium, Journal of the Egyptian Mathematical Society (In Press). http://dx.doi.org/http:10.1016/j.joems.2013.05.008 
Rashidi, M.M., Mohimanian, S.A. and Abbasbandy, S. (2011): Analytic approximate solutions for heat transfer of a micropolar fluid through a porous medium with radiation, Commun Nonlinear Sci Numer Simulat., Vol. 16, No. 4, pp. 1874-1889. http://dx.doi.org/10.1016/j.cnsns.2010.08.016

Rees, D.A.S. and Bassom, A.P. (1996): The Blasius boundary-layer flow of a micropolar fluid, Int. J. Eng. Sci., Vol. 34, No. 1, pp. 113-124. http://dx.doi.org/10.1016/0020-7225(95)00058-5

Yih, K.A. (1999): Effect of radiation on natural convection about a truncated cone, Int. J. Heat Mass Transfer, Vol. 42, No. 23, pp. 4299-4305. http://dx.doi.org/10.1016/S0017-9310(99)00092-7 